IIIIIIIIIIIIIIIIIIIIIIIIIIIIIIIIIIII

Original Article

IIIIIIIIIIIIIIIIIIIIIIIIIIIIIIIIII

\title{
Action mechanism of the novel rice blast fungicide tolprocarb distinct from that of conventional melanin biosynthesis inhibitors
}

\author{
Takahiro Hamada, ${ }^{1, *}$ Mineko Asanagi, ${ }^{2}$ Tomomi Satozawa, ${ }^{3}$ Natsuko Araki, ${ }^{1}$ \\ Shinichi Banba, ${ }^{1}$ Norikazu Higashimura, ${ }^{1}$ Tomohisa Akase ${ }^{1}$ and Kangetsu Hirase ${ }^{1}$ \\ ${ }^{1}$ Agrochemicals Research Center, Mitsui Chemicals Agro, Inc., Mobara, Chiba 297-0017, Japan \\ ${ }^{2}$ Department of Business Innovation Development, Japan Science and Technology Agency, K’s Gobancho, \\ 7 Gobancho, Chiyoda-ku, Tokyo,102-0076, Japan \\ ${ }^{3}$ Purchasing Division, Mitsui Chemicals, Inc., \\ 1-5-2, Higashi-Shimbashi, Minato-ku, Tokyo 105-7117, Japan
}

(Received April 4, 2014; Accepted June 13, 2014)

\begin{abstract}
The target site of tolprocarb, a novel systemic fungicide used for controlling rice blast, was investigated. Tolprocarb decolorized the mycelia of Magnaporthe grisea; the decolorization was reversed by adding scytalone or 1,3,6,8-tetrahydroxynaphthalene $(1,3,6,8-\mathrm{THN})$. This result suggested that the target site of tolprocarb was polyketide synthase (PKS), which regulated polyketide synthesis and pentaketide cyclization in melanin biosynthesis. Further, we produced a transgenic Aspergillus oryzae, which possessed the PKS gene of M. grisea, and performed in vitro assays of PKS using membrane fractions from the transgenic $A$. oryzae. Compared with some conventional melanin biosynthesis inhibitors (cMBIs), tolprocarb only inhibited PKS activity in vitro. These results indicated that tolprocarb's target protein in M. grisea was PKS, which differentiates this fungicide from other cMBIs. () Pesticide Science Society of Japan

Keywords: melanin biosynthesis inhibitors, polyketide synthase, tolprocarb, M. grisea, rice blast.
\end{abstract}

Electronic supplementary materials: The online version of this article contains a supplementary material (Supplemental Table S1), which is available at http://www.jstage.jst.go.jp/browse/jpestics/.

\section{Introduction}

Rice blast caused by Magnaporthe grisea is a major fungal disease affecting rice production worldwide. In its infection process, $M$. grisea produces unicellular infection structures, called appressoria, which adhere tightly to the host surface and produce slender infection pegs that pierce the underlying cell wall of the host. The cell wall of appressoria contains a dense layer of 1,8-dihydroxynaphthalene (1,8-DHN)-melanin synthesized from 1,8-DHN. ${ }^{1)}$ The accumulation of the dark-colored 1,8-DHN-melanin between the plasma membrane and the cell wall is an essential step before the appressoria of Magnaporthe and other fungal species can penetrate host plants. ${ }^{2-9)}$ It is also known that 1,8-DHN-melanin-deficient mutants of M. grisea, which cannot produce the dark gray pigment typical of wildtype mycelia failed to infect intact host plants. ${ }^{10)}$ In various fungal species (e.g., M. grisea, Verticillium dahliae, and Colletotrichum lagenarium), biochemical analysis of enzymes involved

\footnotetext{
* To whom correspondence should be addressed.

E-mail: takahiro.hamada@mitsui-chem.co.jp

Published online August 14, 2014

(c) Pesticide Science Society of Japan
}

in the melanin biosynthetic pathway has been reported. ${ }^{1,8,10-12)}$ The biosynthetic pathway for melanin in fungi starts from pentaketide synthesis and cyclization to form 1,3,6,8-tetrahydroxynaphthalene (1,3,6,8-THN) by polyketide synthase (PKS). The subsequent steps entail reduction of $1,3,6,8$-THN to scytalone, dehydration of scytalone to 1,3,8-trihydroxynaphthalene $(1,3,8$-THN), reduction of $1,3,8$-THN to vermelone, and dehydration of vermelone to 1,8 -dihydroxynaphthalene (1,8-DHN). 1,8 -DHN is then polymerized and oxidized to yield melanin.

A group of compounds that specifically block melanin biosynthesis in the pathogen, here referred to as conventional melanin biosynthesis inhibitors (cMBIs), has long been used for practical control of plant disease. These cMBIs inhibit the penetration of the pathogen into intact host plants by preventing the accumulation of 1,8-DHN-melanin in the appresoria although they barely inhibit mycelial growth, spore germination, and/or appressorial formation of fungi. cMBIs include tricyclazole, ${ }^{2-5)}$ pyroquilon, ${ }^{13)}$ phthalide, ${ }^{5,14,15)}$ carpropamid, ${ }^{16,17)}$ diclocymet, ${ }^{18)}$ and fenoxanil. ${ }^{19)}$ Tricyclazole, pyroquilon, and phthalide inhibit the two reduction steps between 1,3,6,8-THN and scytalone and between 1,3,8-THN and vermelone. ${ }^{3,5,8,14)}$ Carpropamid, diclocymet, and fenoxanil inhibit the two dehydration steps between scytalone and 1,3,8-THN and between vermelone and 
1,8-DHN. ${ }^{16-19)}$ At present, the reduction and dehydration steps are common target sites of cMBIs. However, developing resistance to some cMBIs that inhibit dehydration steps has been a problem in controlling rice blast and other plant diseases. ${ }^{20)}$ For effective disease control, fungicides having different modes of action are required.

Tolprocarb, 2,2,2-trifluoroethyl $N$-[(1S)-2-methyl-1[[(4-methylbenzoyl)amino]methyl]propyl carbamate, which is being developed by Mitsui Chemicals Agro, Inc., has proven highly effective in controlling rice blast. However, tolprocarb's target site has not been revealed. Considering the possibility of cross-resistance with conventional fungicides, it is important to pinpoint this target site. In this report, through recovery tests, tolprocarb's target site was suggested as the polyketide synthesis and/or the pentaketide cyclization steps catalyzed by PKS. Further, we produced transgenic Aspergillus oryzae, which possessed the PKS gene of M. grisea, and using the membrane fraction from the transgenic A. oryzae, we verified that tolprocarb clearly inhibited pentaketide synthesis and/or pentaketide cyclization in melanin biosynthesis.

\section{Materials and Methods}

\section{Chemicals, culture media and strain of $\mathrm{M}$. grisea}

Three fungicides were used in this study: tolprocarb (Fig. 1, purity $99.0 \%$ ), tricyclazole (purity 98.4\%), and carpropamid (purity $98.5 \%$ ). These fungicides and two melanin biosynthesisrelated substances, 1,3,6,8-THN and flaviolin, were synthesized at Agrochemicals Research Center, Mitsui Chemicals Agro, Inc. (Chiba, Japan). Malonyl-CoA was purchased from SigmaAldrich (St. Louis, MO, USA). Potato dextrose agar (PDA) and potato dextrose broth (PDB) were purchased from Kyokuto Seiyaku (Tokyo, Japan) and Difco Laboratories Inc. (Detroit, MI, USA), respectively. Czapek Dox broth and yeast extract were also purchased from Difco. Soluble starch was purchased from Wako (Osaka, Japan). Scytalone was purchased from Indofine Chemical Company, Inc. (Hillsborough, NJ, USA). Restriction enzymes and plasmid vector pBSSK+ were purchased from TAKARA (Osaka, Japan). Fungal expression plasmid pTAex3 was obtained from Professor Katsuya Gomi (Tohoku University, Miyagi, Japan). M. grisea 40901 maintained at Agrochemicals Research Center, Mitsui Chemicals Agro, Inc., was used as a wild-type strain of M. grisea.

\section{Recovery test}

M. grisea 40901 was pre-incubated on a PDA plate to form a mycelial colony. The resultant mycelial colony disc $(5 \mathrm{~mm}$ in

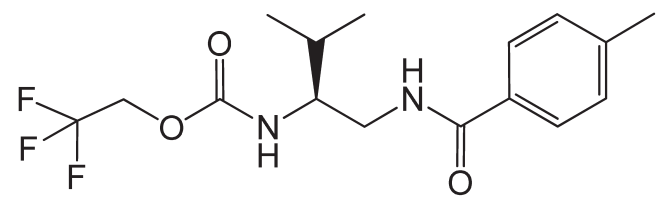

Fig. 1. Structure of tolprocarb. diameter) was removed and inoculated onto fresh PDA plates containing tolprocarb or tricyclazole at $10 \mathrm{ppm}$ and scytalone or $1,3,6,8-\mathrm{THN}$ at $100 \mathrm{ppm}$. The plates were subsequently incubated at $25^{\circ} \mathrm{C}$ for 2 weeks and visually observed for any mycelial color change.

\section{Construction of expression plasmid}

Fungal expression plasmid pTAex 3 possessing the $\alpha$-amylase $(a m y B)$ promoter, terminator of $A$. oryzae, and auxotrophic marker $\operatorname{argB}$ of $A$. nidulans was used. ${ }^{21)}$ A PKS gene of $M$. grisea expression plasmid pTA-pks was constructed on the basis of pUC-pks (Fig. 2). The PKS gene of M. grisea (National Center for Biotechnology Information (NCBI) accession number XP003715434), with an amino acid sequence similar to that of the PKS1 protein of C. lagenarium, was retrieved from NCBI's genetic data base. ${ }^{22}$ ) The four fragments of DNA that covered the whole PKS ORF region were amplified by a polymerase chain reaction (PCR) using DNA of $M$. grisea 40901 maintained at Mitsui Chemicals Agro, Inc., as a template. The primers are mentioned in Supplemental Table S1. These four DNA fragments, designated as pks 5-8, 21-10, 13-12, and 15-14, were digested by appropriate restriction enzymes and then ligated into cloning vector pUC118 DNA Hinc II/BAP (TAKARA, Osaka, Japan), yielding pUC5-8, pUC21-10, pUC13-12, and pUC15-14, respectively. The pUC 5-8 and 21-10 plasmids digested by NcoI/Pst I were ligated into the pUC 5-8 plasmid digested by NcoI/Pst I to produce the plasmid pUC-5' - pks. The pUC15-14 digested by $\mathrm{Xba \textrm {I }} / \mathrm{Pst \textrm {I }}$ was ligated into $\mathrm{pBSSK}+$ digested by $X b a \mathrm{I} / P s t \mathrm{I}$ to produce the plasmid pBS-3'-pks. The pUC-5'-pks digested by PstI/HindIII, the pUC13-12 digested by PstI/SphI, and pBS- $3^{\prime}$-pks digested by SphI/HindIII were all ligated to yield pUC-pks. The pUC-pks digested by EcoRI was ligated into pTAex3 digested by EcoRI to produce pTA-pks. Finally, A. oryzae M-2-3 was transformed with pTA-pks through the protoplast-polyethylene glycol method as described previously. ${ }^{23)}$ As a result, the PKS gene from $M$. grisea comprised 6522 base pairs with an estimated molecular mass of $236 \mathrm{kDa}$.

\section{Extraction of 1,3,6,8-THN from culture medium of transfor- mants}

The transformants appearing on minimal plates after 1 week's incubation were precultured on a PDA plate and inoculated into an induction medium (Czapek Dox broth containing 1\% starch and $0.4 \%$ yeast extract) in a baffled Erlenmeyer flask at $1 \times 10^{4}$ spores $/ \mathrm{mL}$. The transformants were grown for 2 days at $25^{\circ} \mathrm{C}$ on a rotary shaker at $150 \mathrm{rpm}$. The mycelia and culture medium were separated by a Buchner funnel. The culture medium was acidified with $1 \mathrm{~N} \mathrm{HCl}$ and extracted with ethyl acetate for analysis of 1,3,6,8-THN produced by the transformants. The organic phase was desiccated by vacuum drying and dissolved in $50 \mu \mathrm{L}$ of ethanol for HPLC analysis. The HPLC conditions are described later. Mycelia were washed with distilled water and then used for the following cell-free extraction. 


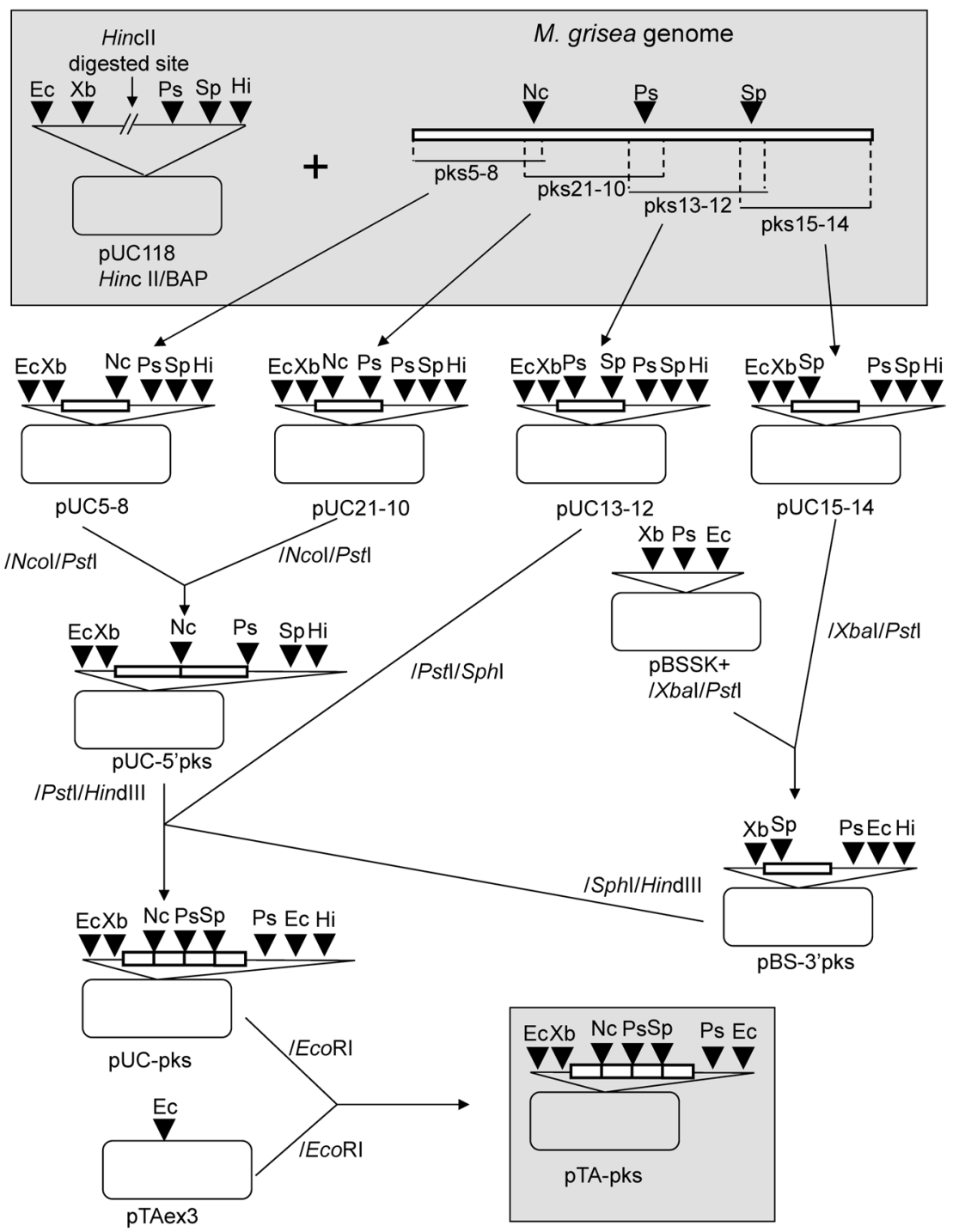

Fig. 2. Structure of pTA-pks and brief outline of its construction process. Ec, EcoRI; Xb, XbaI; Ps, PstI; Sp, SphI; Nc, NcoI; Hi, HindIII; white bar, full length PKS gene of M. grisea.

\section{Cell-free extract preparation}

Cell-free extract preparation was performed by a method described previously. ${ }^{24)}$ Freshly harvested mycelia were flash frozen in liquid nitrogen and pulverized in a mortar with a pestle. The mycelial powder was then suspended in a dilution buffer (50 $\mathrm{mM}$ potassium phosphate buffer, $\mathrm{pH} 7.2$, containing 30\% glycerol, $1 \mathrm{mM}$ dithiothreitol, $1 \mathrm{mM}$ EDTA, and $0.1 \mathrm{mM}$ benzamidine). The mixture was occasionally stirred on ice for $20 \mathrm{~min}$ and subsequently centrifuged at $10,000 \times g$ for $20 \mathrm{~min}$. The supernatant was then filtered through a four-layered gauze to remove residual cell debris (crude cell-free extract). The extract was then centrifuged at $38,900 \times g$ for $80 \mathrm{~min}$. The supernatant was further fractionated by centrifugation at $38,900 \times g$ for $11 \mathrm{hr}$. The precipitate was suspended in a dilution buffer. The precipitate was subjected to SDS-PAGE using the NuPAGE Novex Tris-Acetate Gels system. NuPAGE 3-8\% Tris Acetate Gel with
NuPAGE Tris-Acetate SDS Running Buffer was used for the assay. The gel was stained by SimplyBlue Safestain (Life Technologies, Carlsbad, CA, USA), which indicated the expression of $236 \mathrm{kD}$ of protein. The molecular mass of this protein corresponded well with the deduced molecular mass of PKS. The suspension of precipitate was then used as an enzyme solution in the following experiments.

\section{In vitro assay of $P K S$}

Enzymatic synthesis of 1,3,6,8-THN by PKS was performed by a method described previously. ${ }^{24)}$ A reaction mixture containing $435 \mu \mathrm{L}$ of $50 \mathrm{mM}$ potassium phosphate buffer, $\mathrm{pH} 7.2,15 \mu \mathrm{L}$ of malonyl-CoA solution, $50 \mu \mathrm{L}$ of enzyme solution, and $1 \mu \mathrm{L}$ of tolprocarb or cMBIs was incubated for $1 \mathrm{hr}$ at $25^{\circ} \mathrm{C}$. Final protein concentration in the mixture was $0.91 \mathrm{mg} / \mathrm{mL}$ on average. The reaction mixture was then boiled at $100^{\circ} \mathrm{C}$ for $30 \mathrm{~min}$ for ox- 


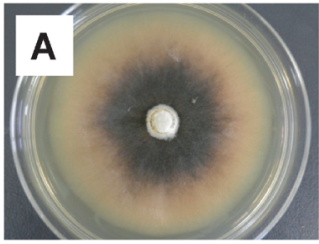

Control

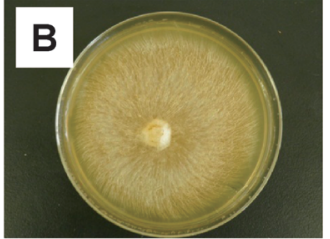

Tolprocarb

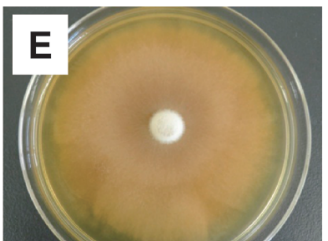

Tricyclazole

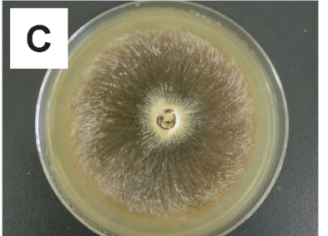

Tolprocarb +scytalone

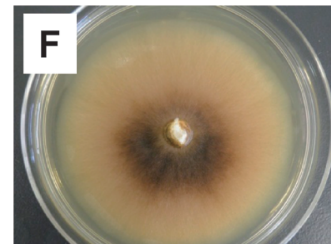

Tricyclazole +scytalone

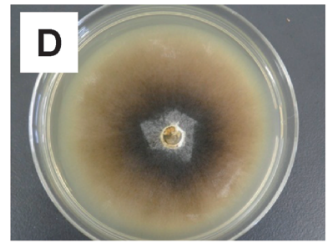

Tolprocarb $+1,3,6,8-\mathrm{THN}$

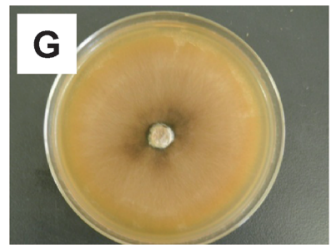

Tricyclazole $+1,3,6,8-\mathrm{THN}$

Fig. 3. Recovery test on M. grisea PDA plate. A, control. Tolprocarb is added at $10 \mathrm{ppm}$ in B, C, and D. Tricyclazole is added at $10 \mathrm{ppm}$ in E, F, and G. Scytalone is added at $100 \mathrm{ppm}$ in C and F. 1,3,6,8-THN is added at $100 \mathrm{ppm}$ in D and G.

idation of $1,3,6,8$-THN to flaviolin and termination of reaction. After acidification with $100 \mu \mathrm{L}$ of $6 \mathrm{M} \mathrm{HCl}$, the reaction mixture was extracted with $500 \mu \mathrm{L}$ of ethyl acetate. The organic phase was desiccated by vacuum drying and dissolved in $50 \mu \mathrm{L}$ of ethanol for HPLC analysis. The 1,3,6,8-THN and flaviolin formed by the enzymatic reaction were identified with LS-MS (date not shown).

\section{HPLC analysis}

Reverse-phase HPLC, used to analyze the 1,3,6,8-THN and flaviolin, was performed under the following conditions: column, ODS-80Ts column $(4.6 \times 150 \mathrm{~mm}$; Tosoh Corp., Tokyo, Japan); column temperature, $25^{\circ} \mathrm{C}$; mobile phase, linear gradient from $5 \% \mathrm{CH}_{3} \mathrm{CN}$ in $\mathrm{H}_{2} \mathrm{O}$ to $50 \% \mathrm{CH}_{3} \mathrm{CN}$ in $\mathrm{H}_{2} \mathrm{O}$ (each contained $2 \%$ acetic acid) over $30 \mathrm{~min}$; flow rate, $1.0 \mathrm{~mL} / \mathrm{min}$; detection, UV detector at $254 \mathrm{~nm}$.

\section{Results}

\section{Recovery test}

The $M$. grisea mycelia became black in a 2-week culture because of melanin formation on an ordinary PDA plate (Fig. 3A), whereas the mycelia became white and orange on a PDA plate with tolprocarb and tricyclazole, respectively (Fig. 3B and E) probably because of melanin biosynthesis inhibition at respective sites on the pathway. The mycelia treated with tolprocarb became black upon addition of scytalone or 1,3,6,8-THN (Fig.

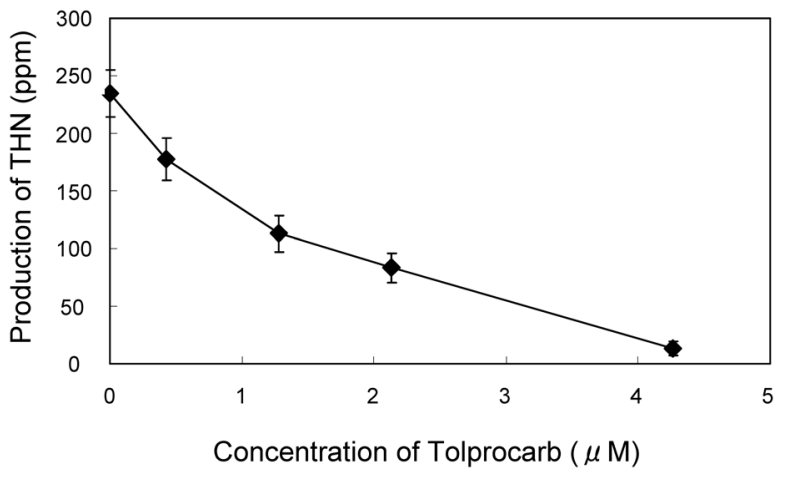

Fig. 4. Effect of tolprocarb on the accumulation of $1,3,6,8-\mathrm{THN}$ in culture of transformant $A$. oryzae. 1,3,6,8-THN is extracted from culture of transformant $A$. oryzae after incubation for $72 \mathrm{hr}$. Average and standard deviations are calculated from three experiments.

3C and D). However, the mycelia treated with tricyclazole did not show any significant change in color upon addition of scytalone or 1,3,6,8-THN (Fig. 3F and G).

2. Effect of tolprocarb on 1,3,6,8-THN accumulation in the culture of A. oryzae transformant

The accumulation of $1,3,6,8$-THN in the culture of $A$. oryzae pTA-pks was examined. Although no accumulation of $1,3,6,8$-THN was detected in the control culture of nontrans- 


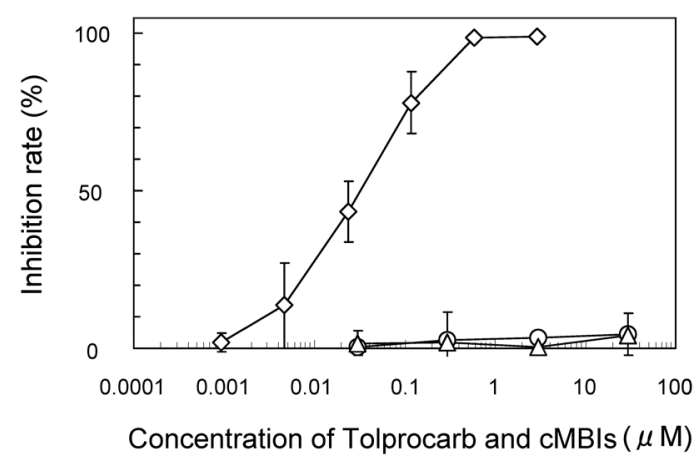

Fig. 5. Effect of tolprocarb and cMBIs on the PKS of M. grisea. PKS inhibition rates by tolprocarb $(\diamond)$, tricyclazole $(\bigcirc)$, and carpropamid $(\triangle)$ are presented. Average and standard deviations are calculated from three experiments.

formant A. oryzae and transformant A. oryzae pTAex3 (data not shown), $234 \mathrm{ppm}$ of $1,3,6,8$-THN was detected in the culture of $A$. oryzae pTA-pks (Fig. 4). In the culture of $A$. oryzae pTApks, accumulation of black pigment was also observed (data not shown). Furthermore, the accumulation of 1,3,6,8-THN and black pigment was reduced depending on the concentration of tolprocarb added to the culture media. The concentration of $1,3,6,8$-THN in the culture was reduced to $13 \mathrm{ppm}$ after adding $4.3 \mu \mathrm{M}$ of tolprocarb (Fig. 4).

\section{Effect of tolprocarb and cMBIs on the PKS of M. grisea}

The enzymatically synthesized $1,3,6,8$-THN was unstable in the reaction mixture, and a part of $1,3,6,8$-THN was rapidly converted to flaviolin. The synthesized $1,3,6,8$-THN was completely converted to flaviolin by heating at $100^{\circ} \mathrm{C}$ for $30 \mathrm{~min}^{24)}$ In this reaction mixture, the PKS product was formed depending on the protein amount and reaction time throughout $1 \mathrm{hr}$; the enzyme reaction then proceeded stably with $\mathrm{pH} 7.2$ at $25^{\circ} \mathrm{C}$, producing $43.8 \mu \mathrm{M}$ of $1,3,6,8-\mathrm{THN}$ in our assay system (data not shown). It was here that we first established the in vitro assay system of $M$. grisea PKS. The PKS inhibition rate increased depending on the increasing concentrations of tolprocarb. The $\mathrm{IC}_{50}$ and $\mathrm{IC}_{90}$ were $0.03 \mu \mathrm{M}$ and $0.23 \mu \mathrm{M}$, respectively; when $0.6 \mu \mathrm{M}$ of tolprocarb was added, the PKS inhibition rate reached $99 \%$ (Fig. 5). On the other hand, PKS was not inhibited when tricyclazole and carpropamid were added, even at $30 \mu \mathrm{M}$ (Fig. 5).

\section{Discussion}

M. grisea causes rice blast, one of the major diseases in rice. In its infection process, unicellular infection structures, called appressoria, and accumulation of 1,8-DHN-melanin in the appressoria are essential for the penetration of $M$. grisea into host plants. ${ }^{1-9)}$ It was reported that 1,8 -DHN-melanin-deficient mutants of $M$. grisea fail to produce the dark gray pigment in mycelia and fail to infect an intact host plant. ${ }^{10)}$ Compounds that inhibit melanin biosynthesis are widely used as practical fungicides for M. grisea. Although cMBIs do not exhibit direct inhibitory activity on fungal growth, these compounds inhibit the accumulation of 1,8-DHN and melanin, which is key to the infection process. However, evolving resistance to some cMBIs that inhibit dehydration steps has become a problem in rice blast control as well as other plant diseases. ${ }^{20)}$ Tolprocarb, which is being developed by Mitsui Chemicals Agro, Inc., showed a high controlling effect on rice blast by both nursery box application and broadcasting on paddy rice (data not shown), and its practical value has been verified. Therefore, in considering the possibility of cross-resistance, clarifying tolprocarb's target site becomes of paramount importance.

Biochemical analysis of enzymes involved in the melanin biosynthetic pathway has been reported in various fungal species (Fig. 6). It is known that accumulation and polymerization of 1,8 -DHN and its intermediates cause pigmentation of the mycelia of M. grisea ${ }^{10)}$ Tricyclazole inhibits the two reduction steps in the biosynthetic pathway of 1,8 -DHN. In a recovery test, tolprocarb decolorized $M$. grisea mycelia, whereas tricyclazole turned them orange (Fig. 3B and E). The mechanism that induces these various color changes is not yet known; a possible explanation is that different sites of inhibition on the pathway entail different compositions of pigments. Addition of 1,3,6,8-THN and scytalone to the medium recovered the black mycelia only when the mycelia were treated with tolprocarb (Fig. 3C and D). These results suggested that tolprocarb's target is at the upper site of scytalone and 1,3,6,8-THN synthesis, or, more precisely, where polyketide synthesis and/or pentaketide cyclization catalyzed by PKS occurs. Although there was another possibility of chemical suppression of PKS expression, here we examined the effect of tolprocarb and cMBIs on the activity of the PKS of M. grisea in the following tests. ${ }^{25)}$

We attempted to examine tolprocarb's effect on PKS from $M$. grisea by establishing an in vitro evaluation system. Because the PKS gene has already been identified in C. lagenarium, ${ }^{22)}$ the PKS gene of $M$. grisea was predicted on the basis of homology with the PKS gene of $C$. lagenarium. As deduced from the amino acid sequence, the PKS protein of $M$. grisea conserves active sites in the following order: $\beta$-ketoacyl synthase (KS), acyltransferase (AT), two acyl carrier proteins (ACP), and thioesterase (TE). This architecture is similar to those of other fungal type I polyketide synthases of Claisen-type cyclization, for example, $C$. lagenarium PKS1, A. nidulans WA, and STCA. ${ }^{26)}$ Because $M$. grisea also produces 1-8-DHN-melanin much like C. lagenarium, it was expected that the PKS gene of $M$. grisea would similarly catalyze the synthesis of 1,3,6,8-THN from malonyl-CoA. ${ }^{24)}$ The PKS gene of $M$. grisea was cloned and subsequently inserted into the pTAex3 plasmid for expression in A. oryzae (Fig. 2). Transformant A. oryzae with pTA-pks showed accumulation of 1,3,6,8-THN (Fig. 4) and black pigments in A. oryzae. This phenomenon suggested that functional PKS of $M$. grisea might be expressed in A. oryzae. Furthermore, as demonstrated by SDSPAGE, a protein having the same size of PKS $(236 \mathrm{kD})$ was expressed in A. oryzae pTA-pks (data not shown). These results indicate that expressed PKS produced $1,3,6,8$-THN in A. ory$z a e$. Because $1,3,6,8$-THN is known to be unstable and easily po- 


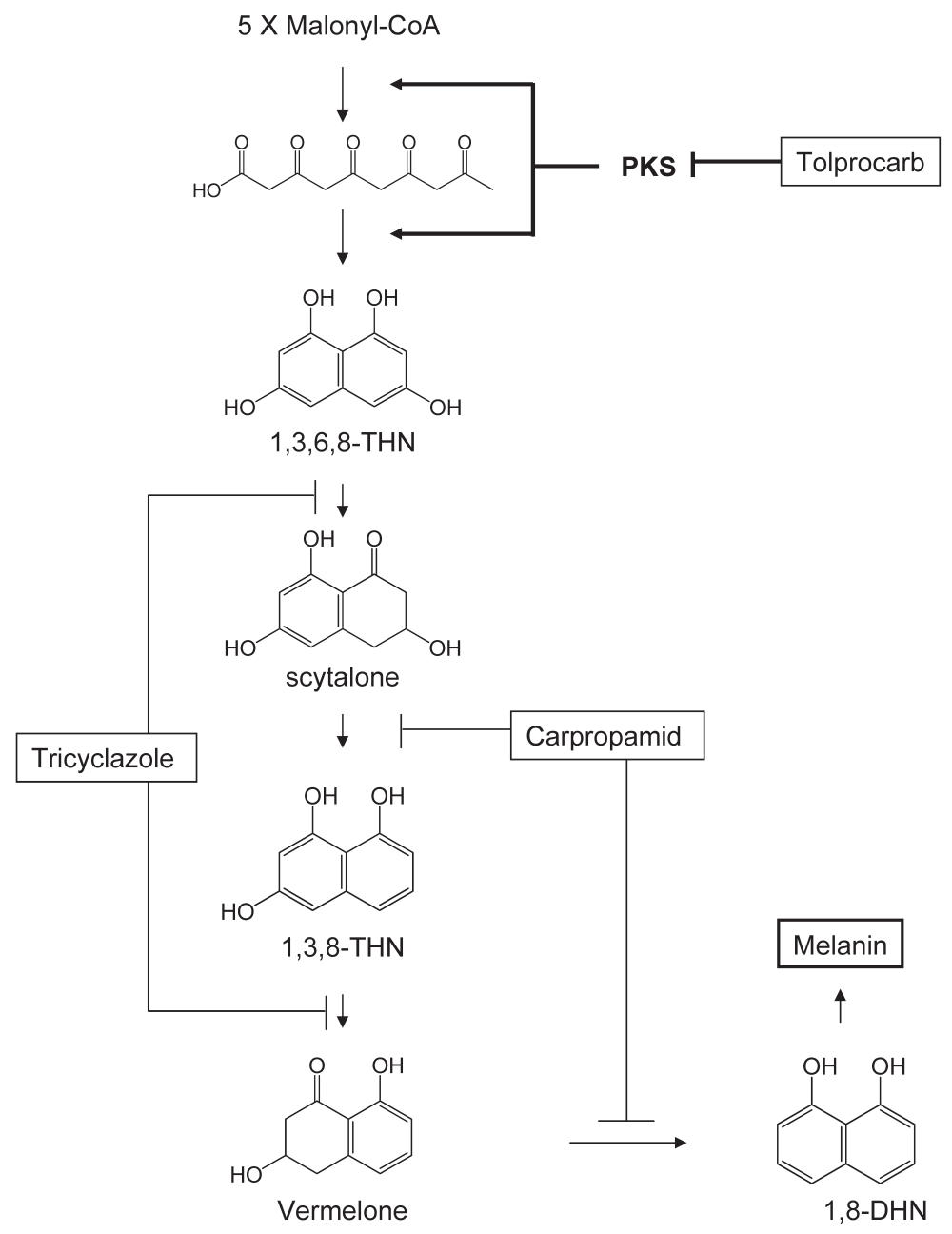

Fig. 6. Melanin biosynthesis pathway and proposed site of action for tolprocarb. PKS protein catalyzes pentaketide synthesis and cyclization steps at the upper site of the pathway. Tolprocarb inhibits PKS as suggested in this study, whereas it is well known that tricyclazole and carpropamid inhibit dehydration and reduction steps, respectively.

lymerized to a black pigment or oxidized to flaviolin, ${ }^{24)}$ it seems logical that a part of $1,3,6,8$-THN would polymerize, causing the accumulation of black pigments. The accumulation of black pigments and THN was reduced by adding tolprocarb (Figs. 3 and 4), suggesting that tolprocarb inhibits PKS.

To confirm direct interaction between tolprocarb and PKS, we assayed PKS activity in a cell-free system. This process was reported by Fujii et al. with the PKS of C. lagenarium ${ }^{24)}$; however, in this study, we achieved an in vitro enzymatic synthesis of $1,3,6,8$-THN from malonyl-CoA by the PKS of $M$. grisea in the cell-free system. This reaction corresponded well with the reaction of C. lagenarium PKS. ${ }^{24)}$ The inhibition study of this system revealed that tolprocarb inhibited the PKS of M. grisea at a $10-100 \mathrm{nM}$ range. On the other hand, tricyclazole and carpropamid did not affect PKS activity (Fig. 5), nor did other cMBIs such as pyroquilon and diclocymet (data not shown). It is therefore suggested that tolprocarb is a novel PKS inhibitor. In other words, although this compound interferes with melanin biosynthesis in M. grisea, the site of action is completely different from that of cMBIs (Fig. 6). Tolprocarb is expected to show no or very low possibility of cross-resistance with cMBIs.

We conducted the same experiments on PKS from both C. lagenarium and $M$. grisea for a comparison because C. lagenarium is insensitive to tolprocarb in antifungal experiments (data not shown). The transgenic A. oryzae that possessed the PKS gene of $C$. lagenarium was created and used in an in vitro assay of PKS using a membrane fraction in the same manner as that for M. grisea. The inhibitory activity of tolprocarb on the PKS of $C$. lagenarium was found to be very low; the $\mathrm{IC}_{90}$ for $C$. lagenarium was more than 100 times higher than that for M. grisea (data not shown). These data support the assertion that PKS is the target protein of tolprocarb.

In conclusion, the target protein of tolprocarb, as opposed to that of cMBIs, was found to be the PKS of M. grisea. It is expected that tolprocarb will prove effective against resistant $M$. grisea because of the fungicide's different target site compared with that of cMBIs. Tolprocarb, $N$-(benzoylamino-alkyl) carbamate, is a new class of fungicide that features a novel mode of 
action among agrochemicals.

\section{Acknowledgements}

We express our sincere gratitude to Professor Katsuya Gomi (Tohoku University) for providing the expression plasmid.

\section{References}

1) A. A. Bell and M. H. Wheeler: Annu. Rev. Phytopathol. 24, 411-451 (1986).

2) T. Okuno, K. Matsuura and I. Furusawa: J. Pestic. Sci. 8, 357-360 (1983).

3) C. P. Woloshuk and H. D. Sisler: J. Pestic. Sci. 7, 161-166 (1982).

4) C. P. Wolshuk, H. D. Sisler, M. C. Tokousbalides and S. R. Dutky: Pestic. Biochem. Physiol. 14, 256-264 (1980).

5) I. Yamaguchi, S. Sekido and T. Misato: J. Pestic. Sci. 7, 523-529 (1982).

6) Y. Kubo, K. Suzuki, I. Furusawa, N. Ishida and M. Yamamoto: Phytopathol. 72, 498-501 (1982).

7) Y. Kubo, K. Suzuki, I. Furusawa and M. Yamamoto: Exp. Mycol. 7, 208-215 (1983).

8) Y. Kubo, K. Suzuki, I. Furusawa and M. Yamamoto: Pestic. Biochem. Physiol. 23, 47-55 (1985).

9) K. Suzuki, Y. Kubo, I. Furusawa, N. Ishida and M. Yamamoto: Can. J. Microbiol. 28, 1210-1213 (1982).

10) F. G. Chumley and B. Valent: Mol. Plant Microbe Interact. 3, 135-143 (1990).

11) M. H. Wheeler: Exp. Mycol. 6, 171-179 (1982).
12) R. D. Stipanovic and A. A. Bell: J. Org. Chem. 41, 2468-2469 (1976).

13) C. P. Wolshuk, P. M. Wolkow and H. D. Sisler: Pectic. Sci. 12, 86-90 (1981).

14) T. Chida and H. D. Sisler: J. Pestic. Sci. 12, 49-55 (1987).

15) S. Inoue, K. Maeda, T. Uematsu and T. Kato: J. Pestic. Sci. 9, 731-736 (1984).

16) Y. Kurahashi, T. Hattori, S. Kagabu and R. Pontzen: Pestic. Sci. 47, 199-202 (1996).

17) Y. Kurahashi, S. Sakawa, T. Kinbara, K. Tanaka and S. Kagabu: J. Pestic. Sci. 22, 108-112 (1997).

18) A. Manabe, K. Maeda, M. Enomoto, H. Takano, T. Katoh, Y. Yamada and Y. Oguri: J. Pestic. Sci. 27, 257-266 (2002).

19) A. Nishimura and I. Hino: Agrochem. Jpn. 81, 13-15 (2002).

20) F. Suzuki, J. Yamaguchi, A. Koba, T. Nakajima and M. Arai: Plant Dis. 94, 329-334 (2010)

21) T. Fujii, H. Yamaoka, K. Gomi, K. Kitamoto and C. Kuagai: Biosci. Biotechnol. Biochem. 59, 1869-1874 (1995).

22) Y. Takano, Y. Kubo, K. Shimizu, K. Mise, T. Okuno and I. Furusawa: Mol. Gen. Genet. 249, 162-167 (1995).

23) K. Gomi, Y. Iimura and S. Hara: Agric. Biol. Chem. 51, 2549-2555 (1987).

24) I. Fujii, Y. Mori, A. Watanabe, Y. Kubo, G. Tsuji and Y. Ebizuka: Biochem. 39, 8853-8858 (2000).

25) S. Okamoto, M. Sakurada, Y. Kubo, G. Tsuji, I. Fujii, Y. Ebizuka, M. Ono, H. Nagasawa and S. Sakuda: Microbiol. 147, 2623-2628 (2001).

26) I. Fujii, A. Watanabe, Y. Mori and Y. Ebizuka: Actinomycetol. 12, 1-14 (1998). 